\title{
A Rare Case of an Ascending Aorta and Aortic Arch Aneurysm with an Aberrant Right Common Carotid Artery and a Proximal Descending Aortic Ectasia
}

\author{
Mikhail M Olalo, MD* and Syril Bren P Guillermo, MD, FPCP, FPCC \\ Department of Internal Medicine, Southern Philippines Medical Center, Philippines
}

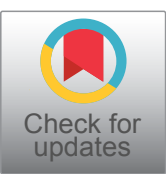

*Corresponding author: Mikhail M Olalo, MD, Department of Internal Medicine, Southern Philippines Medical Center, Philippines

\begin{abstract}
Ascending aortic aneurysms are asymptomatic and are usually discovered as an incidental finding on chest imaging. However, larger aneurysms can present with symptoms resulting from compression of surrounding structures including the trachea, bronchi, and the esophagus which can result in hoarseness, cough chest pain or back pain.

The presence of an aortic arch anomaly, specifically an aberrant right common carotid artery, in a background of an aortic arch aneurysm is extremely rare with a worldwide incidence of $<1 \%$. They are usually asymptomatic but can result to catastrophic life-threatening events and pose significant challenges to surgical or endovascular treatment.

This is a case of a 63-year-old Filipino male who presented with a sudden onset of dull back pain radiating to the left anterior chest. Workup revealed an ascending and aortic arch aneurysm with an aberrant right common carotid artery arising directly from the transverse aorta. Surgical aortic arch debranching was done to repair the aberrant vessels prior to Thoracic Endovascular Aortic Repair (TEVAR) wherein a custom-made Thoracic Valiant graft was deployed on the aneurysm. The patient was discharged on the $4^{\text {th }}$ day after TEVAR without any complaints of dyspnea, back pain nor chest pain with no neurologic and visceral organ dysfunction.

This case has emphasized that knowledge on the anatomy of the aortic arch is imperative in planning out thoracic surgery and endovascular interventions especially on rare anatomic anomalies such as seen in this case.
\end{abstract}

\section{Keywords}

Aortic aneurysm, Aberrant right common carotid artery, TEVAR

\section{Objectives}

This case report aims to:

1. Present a case of a 63-year-old male complaining of sudden onset of severe dull back pain;

2. Discuss the approach and management of the case; and

3. Present the incidence and prognosis of the case.

\section{Introduction}

Ascending aortic aneurysm is the dilatation of the aortic vessel to more than $150 \%$ of the diameter expected for sex, age, and body weight while lesser dilatations are called ectasia. It is most often noted as an incidental finding on routine imaging. It can present as a challenge to physicians because they are often asymptomatic until they present with dissection, expansion or rupture.

An aortic aneurysm becomes a life-threatening condition with rupture and dissection leading to significant short and long-term morbidity and mortality. The incidence increases with age and the prevalence has been estimated to be increasing over the years.

The Centers for Disease Control and Prevention (CDC) reports that the incidence of ascending thoracic aortic aneurysm (TAA) is approximately 10 per 100,000 person-years. Women and men have similar incidences of thoracic aortic aneurysm but the age at diagnosis is a decade higher in women (70s) than in men (60s) [1].

Aortic arch anomalies on an ascending or aortic arch 
aneurysm are rarely documented. One anomaly is an aberrant right common carotid artery with a reported prevalence of $<1 \%$ in autopsy series and $<1 \%$ in 4 -vessel aortic arch anomalies. The exact natural history and overall prognosis are unknown due to its rarity which often pose significant challenges to surgical and endovascular treatment.

\section{Case Protocol}

$\mathrm{CH}$, a 63-year-old male, married, pastor from Midsayap, Cotabato City was admitted in our institution due to a sudden onset of severe dull back pain.

Five years PTA, the patient experienced recurrent episodes of back pain which was dull in character, non-radiating, with a pain scale of $2 / 10$, not aggravated by physical exertion. This was associated with occasional tolerable dysphagia on solid food. The patient tolerated symptoms. No consultation was done and no medication was taken.

Four days prior to admission, the patient was just sitting and had sudden onset of dull back pain with a pain scale $7 / 10$ radiating to the left anterior chest. This was not associated with any dyspnea, diaphoresis, syncope or any palpitations. It was not aggravated and relieved with any position changes. The patient immediately sought consultation in a local hospital. Chest X-ray of the patient revealed a widened mediastinum where he was managed as a case of thoracic aneurysm, hypertension stage II. The patient was then referred in our institution for further evaluation and management.

The patient is a known hypertensive for 23 years maintained on losartan $50 \mathrm{mg}+$ hydrochlorothiazide $12.5 \mathrm{mg} / \mathrm{tab} 1 \mathrm{tab}$ once a day with poor compliance. His usual blood pressure is $130 / 80 \mathrm{mmHg}$, with a highest recorded blood pressure at $150 / 100 \mathrm{mmHg}$. He is non-diabetic with no history of myocardial infarction, cerebrovascular accident or any pulmonary tuberculosis. He has no known food and drug allergy.

Family history revealed hypertension on the paternal side with a history of myocardial infarction on his sister at the age of 53-years-old.

He is a smoker for 15 pack years and a previous alcoholic beverage drinker, both hard liquor and beer, approximately three to four times a week, averages 300 $\mathrm{mL}$ per drinking session. Hedenies of any illicit drug use. $\mathrm{He}$ is a Protestant and works as a pastor with an active lifestyle.

Review of systems revealed absence of any easy bruising, palpitations, chest pain on exertion, orthopnea nor any paroxysmal nocturnal dyspnea.

Relevant vital signs include blood pressure of $160 / 100 \mathrm{mmHg}$ on all extremities and a body mass index of 31.2 (obese). Pertinent physical examination findings revolved around the cardiovascular system. Carot-

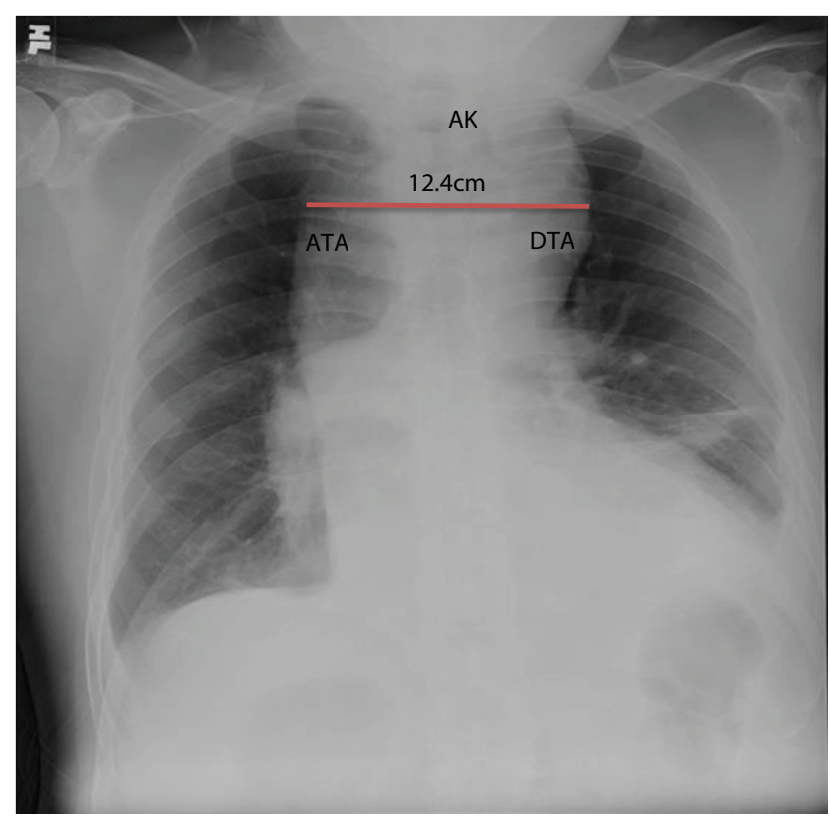

Figure 1: Chest Radiography. There is widening of the mediastinum by about 12.4 centimeters in its widest diameter. ATA: Ascending thoracic aorta; AK: Aortic knob; DTA: Descending thoracic aorta.

id pulses were full with no noted bruit. Jugular venous pressure was at $8 \mathrm{~cm}$ water at a 30 -degree angle. The precordium was adynamic with absent palpable heaves nor thrills. The point of maximal impulse was displaced to the left $6^{\text {th }}$ intercostal space, anterior axillary line with a normal heart rate at 66 beats per minute with a regular rhythm. No murmurs were appreciated. Abdominal findings revealed absence of any pulsatile mass. Glasgow coma scale was 15 with no focal neurologic deficit. Ancillary laboratory tests revealed a normal Troponin I of $0.02 \mathrm{ng} / \mathrm{ml}$ (Appendix A). Chest radiography (Figure 1) revealed a widened mediastinum compatible with a thoracic aortic aneurysm.

The 12-lead electrocardiogram (Figure 2) showed sinus rhythm, left ventricular hypertrophy, isolated premature ventricular contraction, non-specific T-wave changes and QRS alternans.

The patient was admitted as a case of a thoracic aortic aneurysm and was started with amlodipine $5 \mathrm{mg} / \mathrm{tab}$ 1 tab once a day, losartan $100 \mathrm{mg} / \mathrm{tab} 1$ tab once a day, carvedilol $12.5 \mathrm{mg} / \mathrm{tab} 1 \mathrm{tab}$ twice a day, atorvastatin $80 \mathrm{mg} / \mathrm{tab} 1$ tab once a day at bedtime with a goal of maintaining systolic blood pressure at $100-110 \mathrm{mmHg}$ and a heart rate of 55 to 65 beats per minute.

$\mathrm{CT}$ angiogram (Figure 3) was done revealing an anomalous origin of the right subclavian artery, right common carotid artery, and left common carotid artery, with an absent brachiocephalic trunk. An ascending aorta and aortic arch aneurysm, and proximal descending aorta ectasia with perianeurysmal hematoma; atherosclerotic and tortuous aorta were noted. 


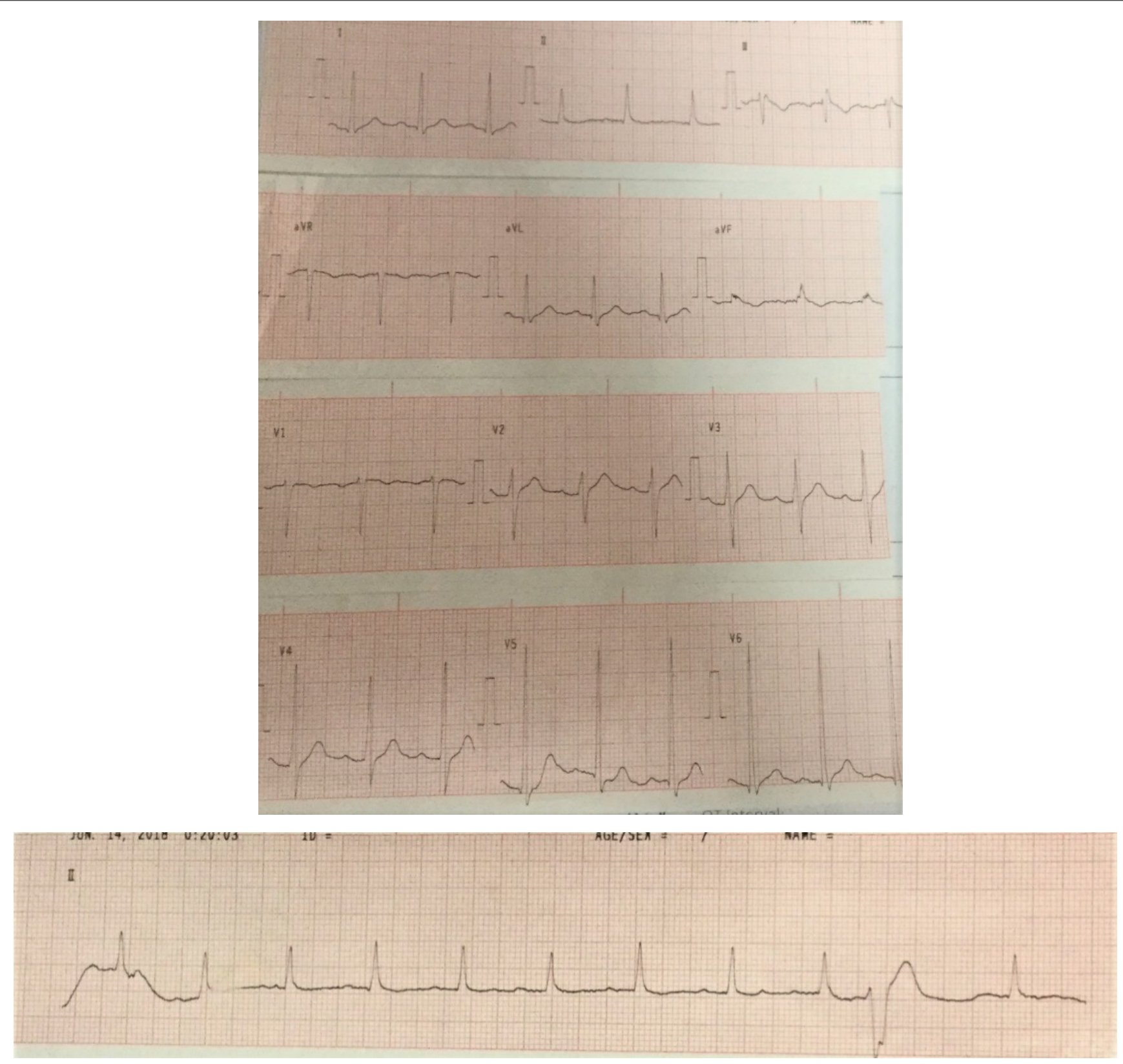

Figure 2: 12-lead electrocardiogram.

2D Echo was done showing normal left ventricular size with hypertrophied walls with hypokinesia of the anterior interventricular septum from base to mid segment. The left ventricular ejection fraction is $57 \%$. Right ventricle is normal with a good contractility and a normal left and right atrium. There is a moderate mitral regurgitation and a mild tricuspid regurgitation with a normal pulmonary systemic pressure.

Carotid duplex scan shows type III plaques in the bilateral carotid bulbs and bilateral proximal to distal common carotid arteries without significant hemodynamic disturbances. There is $1-15 \%$ stenosis in the bilateral carotid bulbs, $<50 \%$ stenosis in the bilateral proximal to distal common carotid arteries, normal anterograde flow in the bilateral vertebral arteries and increased common carotid artery-intima media thickness.

Coronary angiogram (Figure 4) revealed a severe single vessel coronary artery disease involving the left anterior descending artery (LAD).

Conventional CT aortogram (Figure 5) with 3D reconstruction (Figure 6) was done for detailed surgical anatomy with the involved aberrant vessels. There was a noted aberrant takeoff of the right common carotid artery arising directly from the transverse aorta separated from the right subclavian artery, which is positioned proximal to the subclavian artery. The left common carotid arises after the left subclavian artery. It is normal in caliber with no stenosis demonstrated.

The patient first underwent aortic arch debranching (Figure 7 and Figure 8). This is done to have an optimal placement of the proximal end of the endovascular stent graft in the landing zone which is the most critical step in the endovascular part of the intervention.

A repeat $C T$ angiogram of the thoracic and abdominal aorta post-operatively (Figure 9) revealed an intact and 

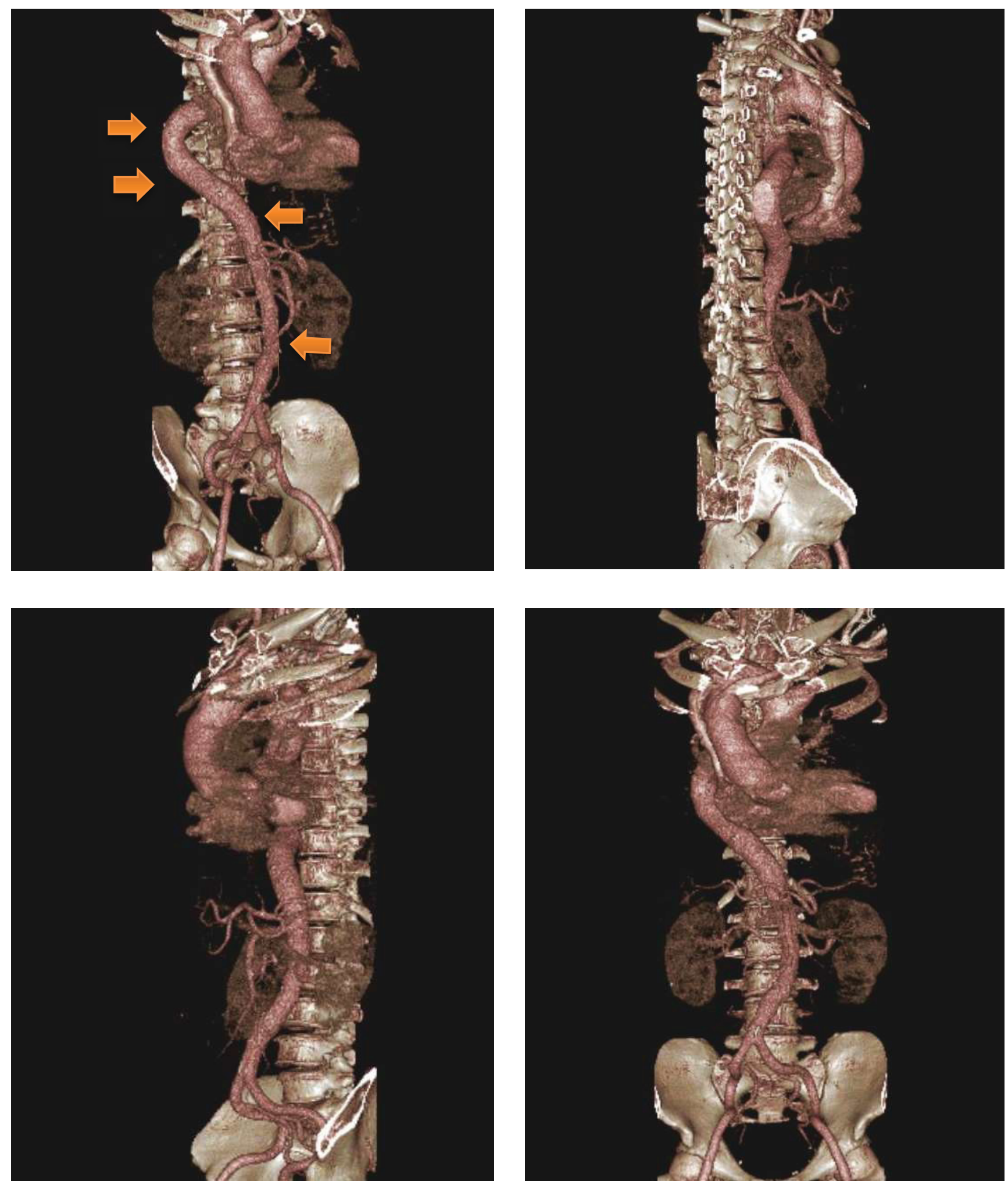

Figure 3: CT angiogram. Results show few scattered mural calcifications in the wall of the aorta which is tortuous (orange arrowhead), crossing the midline at the level of T7 and descending in the right paravertebral region until the body of T10, where it then crosses the midline back to the left paravertebral area. Contrast study shows opacification of the ascending aorta down to both iliac arteries. Four aortic arch branches are identified, with the right subclavian and common carotid arteries arising directly from the aorta. There is no identified brachiocephalic trunk.

patent aortic graft, transplanted right and left common carotids and left subclavian-carotid graft anastomosis; thrombosed right subclavian-aortic graft anastomosis.

Two weeks post-operatively, the patient underwent Thoracic Endovascular Aortic Repairof the aneurysm (Figure 10). A custom-made endovascular graft was manufactured based on computer modeling of the CT images.

The patient was discharged improved on the $4^{\text {th }}$ day post-TEVAR without any neurological and visceral organ dysfunction. There were no complaints of dyspnea, back pain nor chest pain. Maintenance medications in- 
cluded Irbesartan, Carvedilol and Amlodipine for strict blood pressure control.

\section{Discussion}

An aneurysm is typically defined as an increase in size of more than $50 \%$ above the normal arterial diameter. It can occur anywhere along the aorta - from the aortic root to the bifurcation. Majority of these aneurysms are degenerative in nature such as in our patient. Other less common causes are associated with infection such as a mycotic infection, inflammation and connective tissue disease [2]. TAAs are classified into aortic root or ascending aortic aneurysms (60\%), followed by

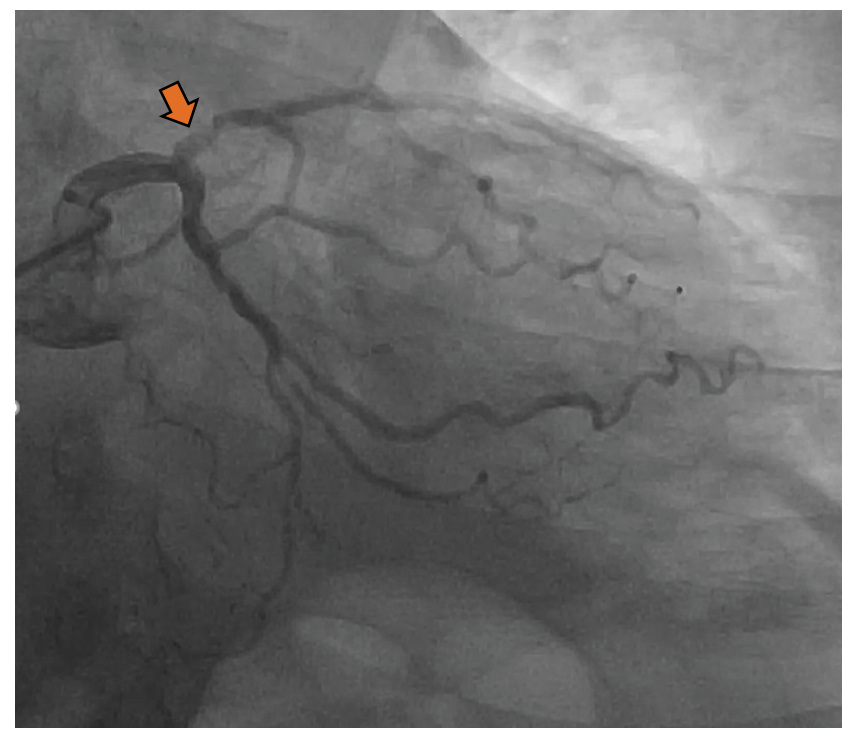

Figure 4: Coronary Angiogram. Six mm normal-sized left main coronary artery is noted. However, the LAD had $3.5 \mathrm{~mm}$ vessel with $70 \%$ proximal eccentric stenosis (arrowhead) before D1 which is a good sized vessel with intimal irregularities. The rest of the LAD has intimal irregularities. aneurysms of the descending aorta (35\%) and aortic $\operatorname{arch}(10 \%)$ [3].

Aneurysms are usually silent unless an imaging study happens to have been performed; thus, true incidence is hard to estimate. Lethal thoracic aortic dissections are also often misdiagnosed as myocardial infarctions [4]. A local study reported that ruptured aortic aneurysm has a higher mortality rate than myocardial infarction (MI) wherein 75 to 80 percent with ruptured aortic aneurysm die compared to 10 to 20 percent of people coming with MI.

Sudden onset of severe chest and back pain is the most typical symptom of an acute aortic syndrome. The pain may be described as sharp, ripping, tearing, or knife-like and is usually different from other causes of chest pain. The abruptness of its onset is the most specific characteristic [5]. Minor and less specific symptoms such as chronic back pain which results from direct pressure on nearby structures or by distension of visceral organs which was the presenting symptom of the patient.

Differential diagnoses of the patient's case aside from an aortic aneurysm include musculoskeletal pain, pulmonary embolism, pericarditis and an acute coronary syndrome. Musculoskeletal pain can easily be ruled out because of the absence of any reproducible pain on palpation of the affected area and the pain is not provoked by posture, motion or pressure. Pulmonary embolism can be considered because it can occasionally present with back pain and sudden onset of dyspnea. However, because of the absence of hypoxia and the typical pleuritic chest pain, deep vein thrombosis, calf swelling or tenderness makes the diagnosis unlikely. Pericarditis can also be considered because of the onset of chest
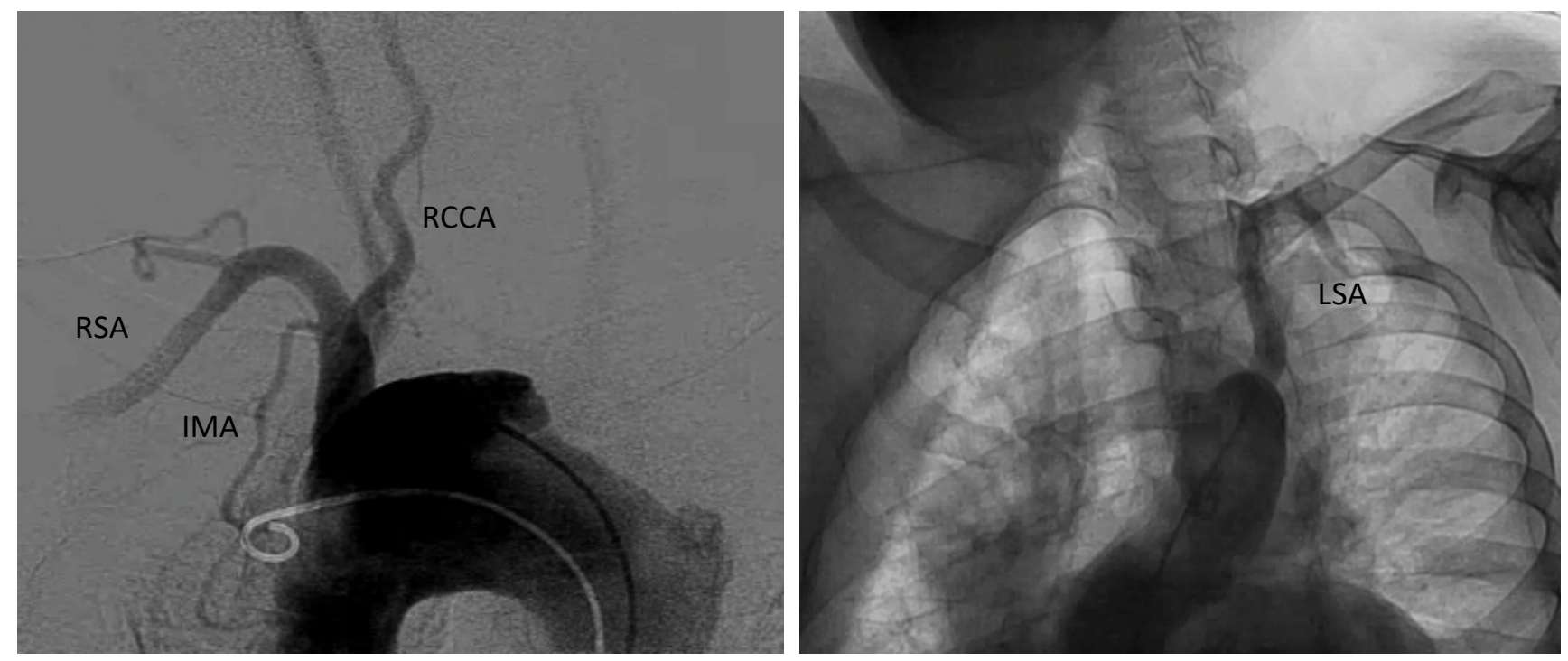

Figure 5: Conventional CT Aortogram. Results revealed a severely atherosclerotic and tortuous thoracic aorta with large ulcer appreciated in the transverse segment, a patent and normal caliber right common carotid artery, right subclavian artery, and left subclavian artery. An aberrant origin of the right common carotid artery arising directly from the transverse aorta is incidentally noted. RSA: Right subclavian artery; IMA: Internal mammary artery; RCCA: Right common carotid artery; LSA: Left subclavian artery. 

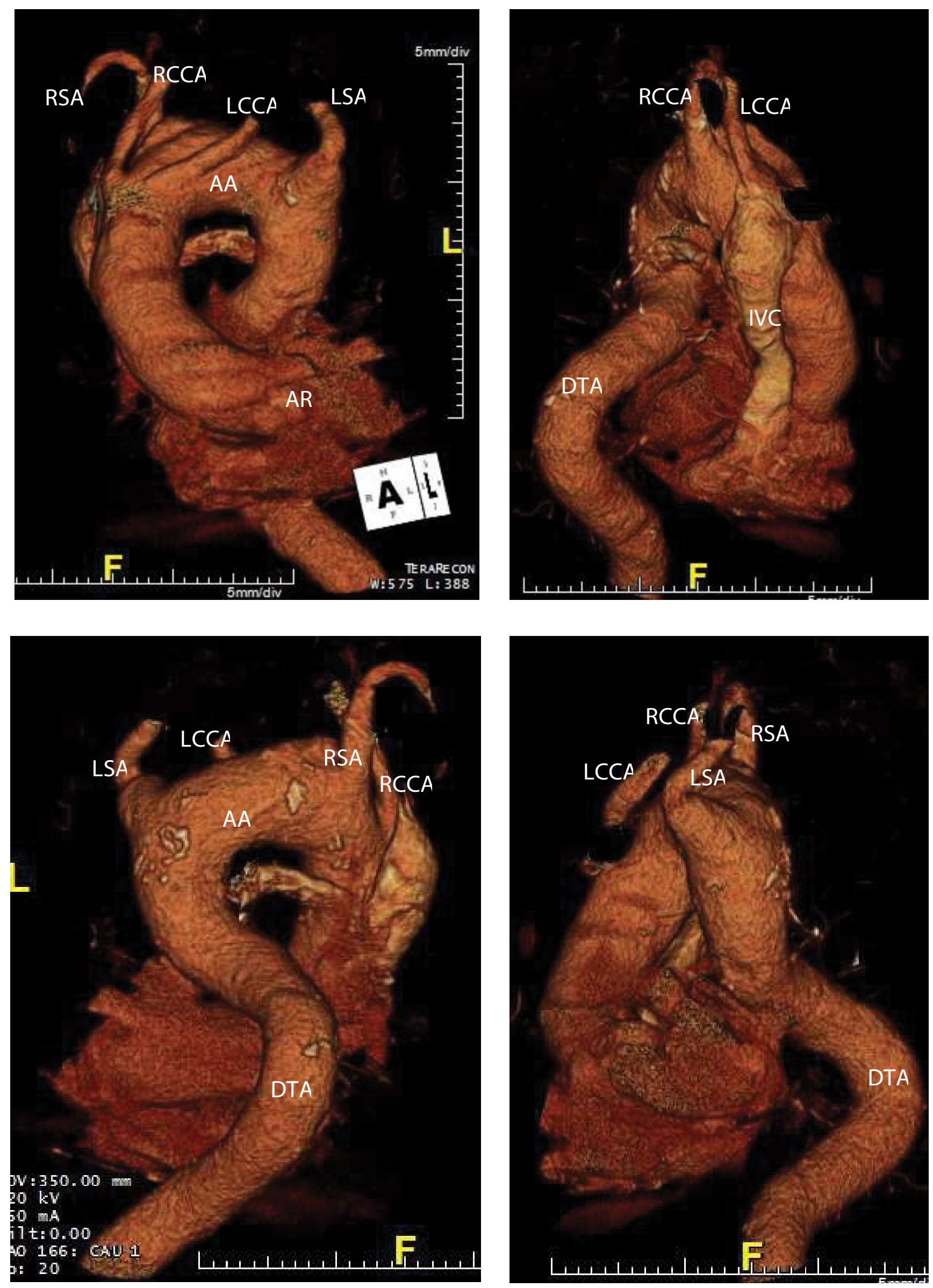

Figure 6: 3D Reconstruction of CT Aortogram (Pre-operative). LSA: Left Subclavian Artery; LCCA: Left Common Carotid Artery; RSA: Right Subclavian Artery; RCCA: Right Common Carotid Artery; DTA: Descending Thoracic Aorta; AA: Aortic Arch; IVC: Inferior Vena Cava. 

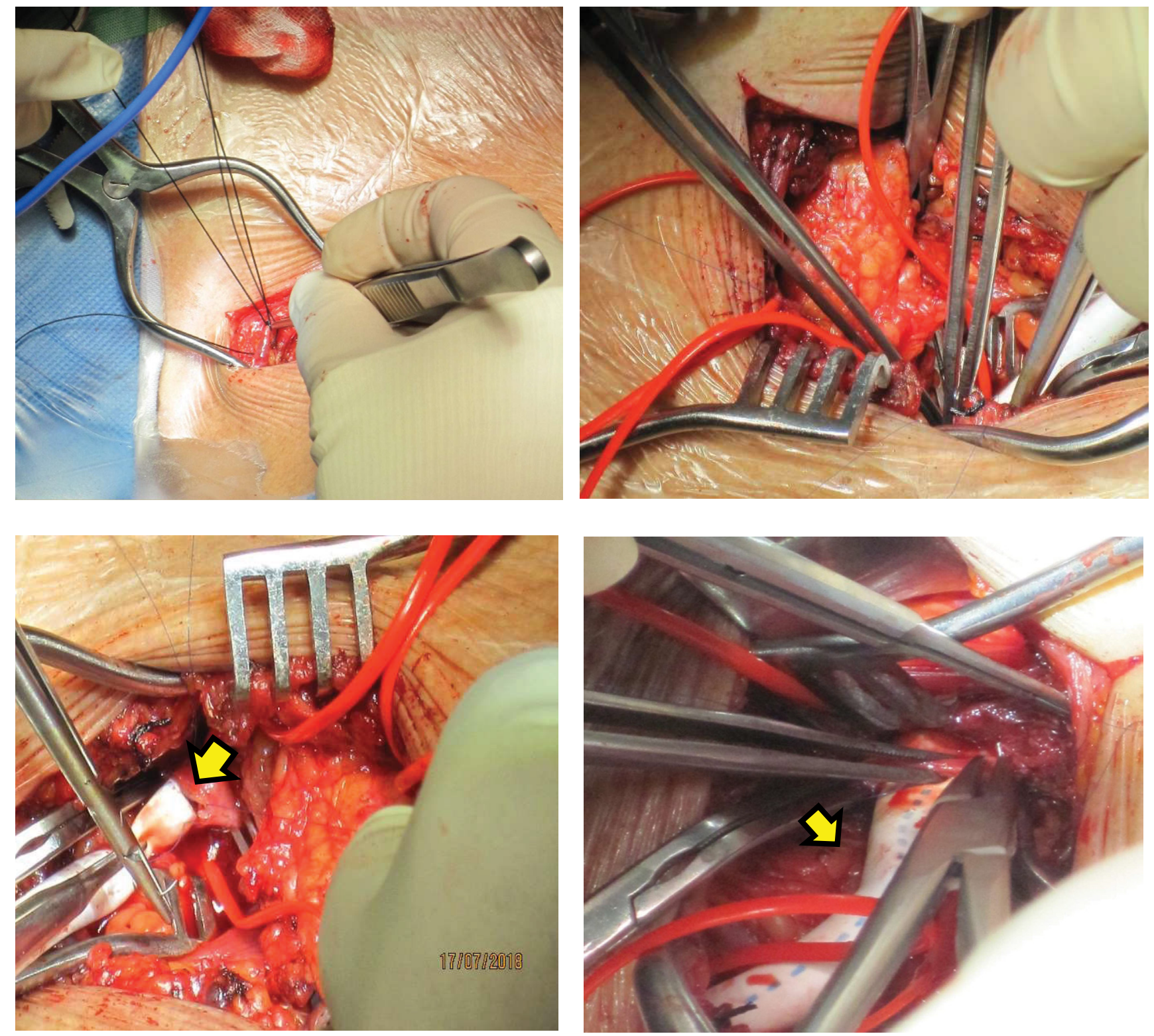

Figure 7: Intraoperative pictures of aortic arch debranching using a Polytetrafluoroethylene graft (arrowhead) for the anastomosis.
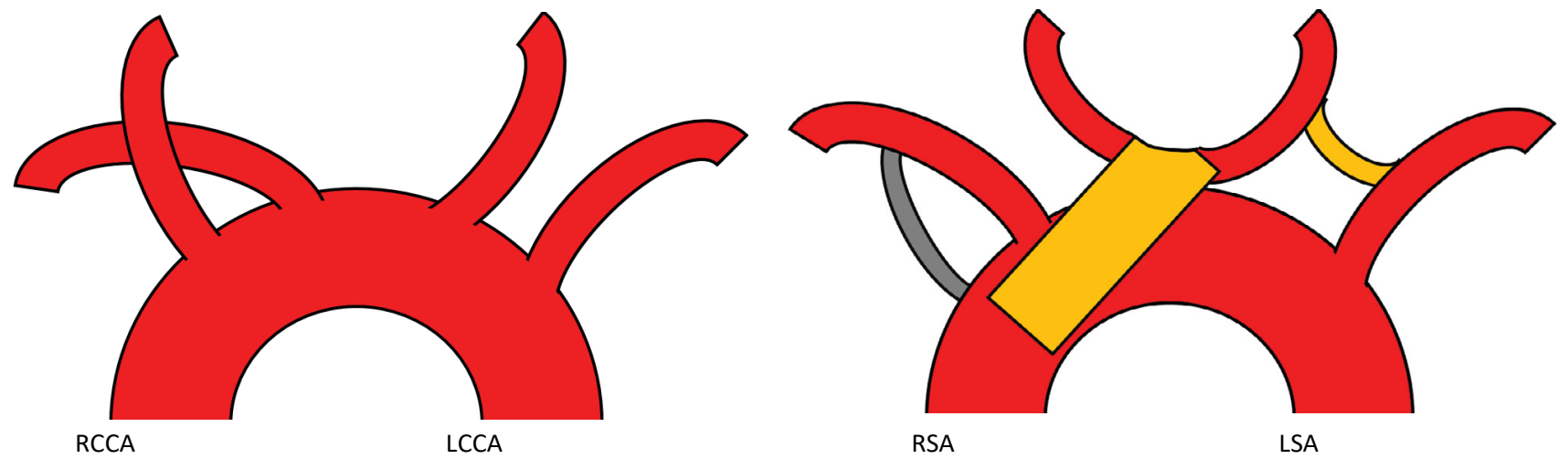

Figure 8: Pre-operative and post-operative aortic arch debranching. Complex thoracic aortic graft implantation and reimplantation of bilateral carotid arteries $[\mathrm{A}]$, carotico-left subclavian artery graft anastomosis [B], right subclavian arteryaortic graft anastomosis [C].

pain however it is considered unlikely because it was not aggravated or relieved with any position changes typical of acute pericarditis. Acute coronary syndrome can be highly considered because of the onset of left sided chest pain however ECG and cardiac biomarkers are needed to fully establish the diagnosis. 

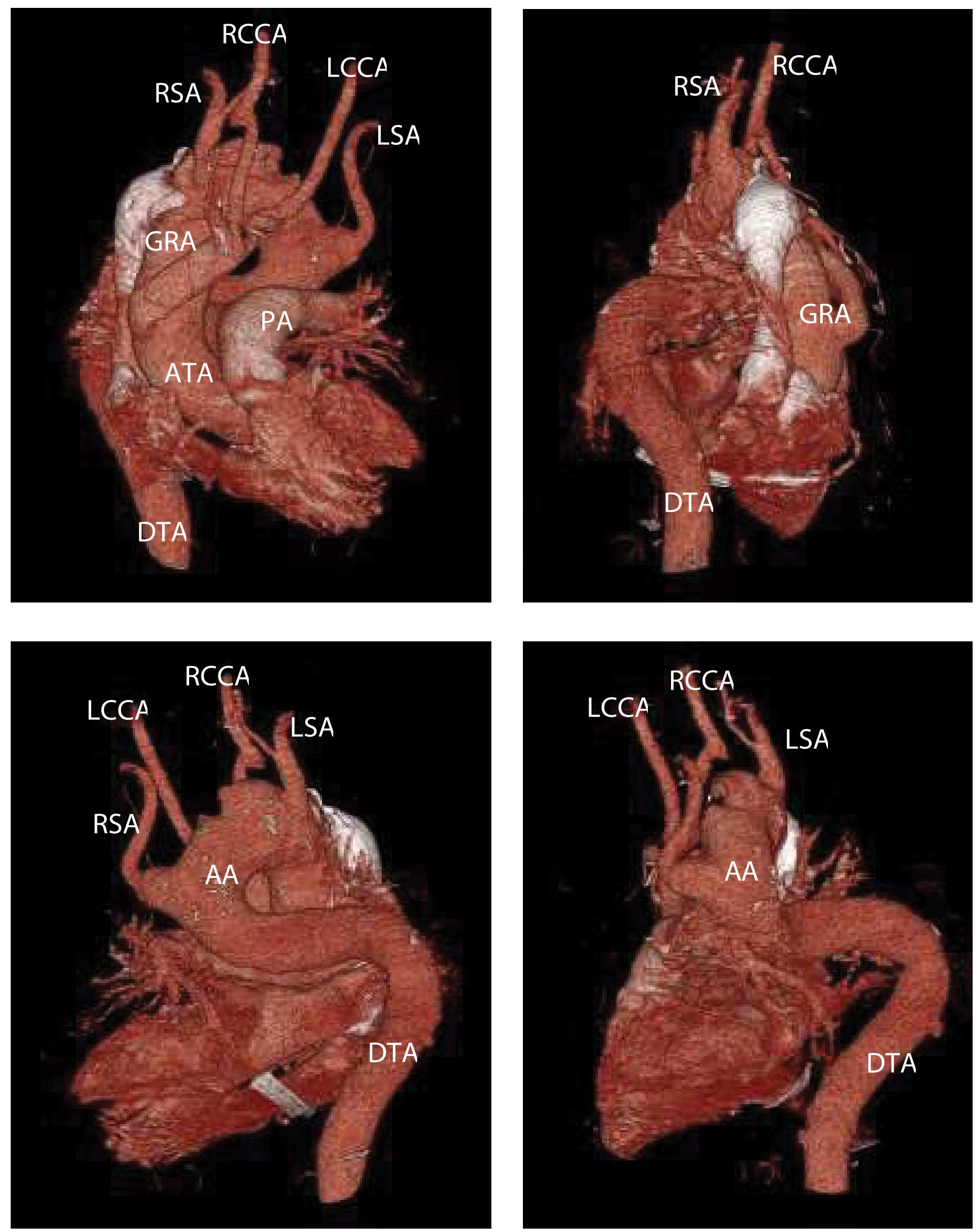

Figure 9: 3D Reconstruction of CT aortogram (Post-operative). Results showed an aortic graft (GRA) arising from the proximal ascending aorta which is intact and patent. The transplanted right and left common carotid arteries to the aortic graft are intact and patent with no significant stenosis demonstrated. The left subclavian-left carotid graft is intact and patent. The aortic graft-right subclavian graft anastomosis is thrombosed with no opacification.

Prompt diagnosis is important in patients with an acute aortic syndrome [2]. A chest radiograph shows widening of the mediastinal shadow as seen in our patient. It may also show displacement or compression of the trachea or left main stem bronchus. Computed tomography (CT) is the most reliable diagnostic tool in patients with acute aortic dissection or ruptured aortic aneurysm. Arteriography is not a substitute [6], although 

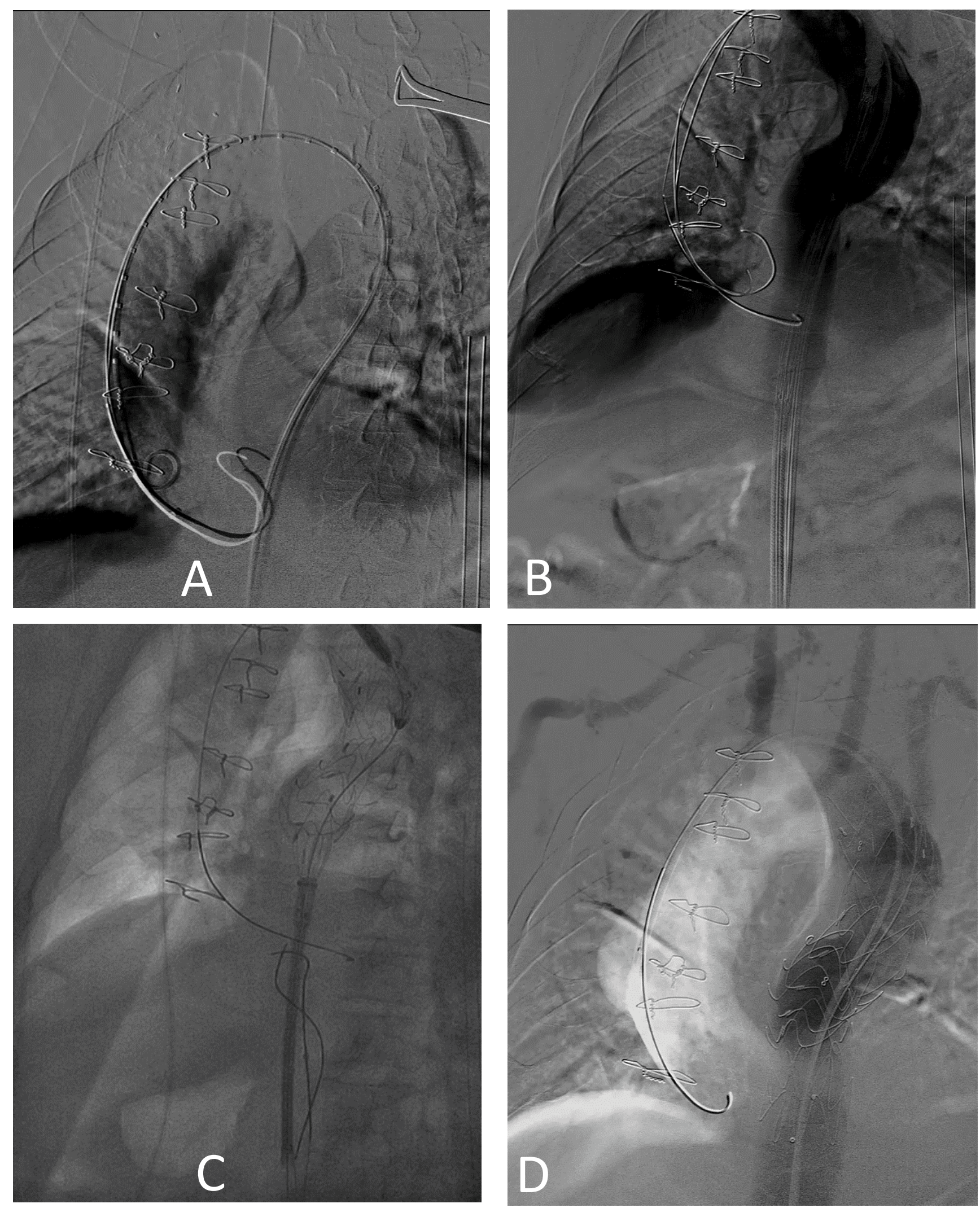

Figure 10: Thoracic Endovascular Aortic Repair (TEVAR). Series of images showing the deployment of the custom-made graft. A French 26 introducer sheath $[A, B]$ was put in place at the left femoral artery. A Thoracic Valiant $40 / 36 \mathrm{~mm} \times 150 \mathrm{~mm}$ stent [C] over a back-up Meier stiff wire was deployed above the left subclavian artery. Post-stent deployment showed patent graft [D], no endoleaks and no dissection noted.

angiography is appropriate for surgical planning.

Two-dimensional echocardiography, and particularly transesophageal echocardiography, can be used to assess the proximal ascending aorta and descending tho- racic aorta. Contrast aortography is frequently required preoperatively to assess the length of the aneurysm and involvement of branch vessels.

Routine work up for surgery in our patient revealed 
a congenital aortic anomaly. Normally, the aortic arch is almost always left sided and gives rise to the three great vessels; first the brachiocephalic trunk (BCT), then the left common carotid artery, and finally the left subclavian artery. The BCT branches into right subclavian artery and right common carotid artery. This branching pattern occurs in $64.9-94.3 \%$ of cases and is described as "normal" [7].

In our patient, an aberrant right common carotid artery was incidentally noted on the work-up of the aneurysm. It has an estimated prevalence of $<1 \%$ in 4 -vessel aortic arch anomalies. It is uncommon and a result from an aberrant development of one or more components of the embryonic pharyngeal arch system. The variations of the aortic arch can be explained by persistence of segments of the aortic arches that normally regress or the disappearance of segments that normally remain $[8,9]$.

The initial management of our patient included the control of the heart rate and blood pressure to slow down the expansion rate and reduce the risk of dissection or rupture $[10,11]$. Patients with aortic aneurysm should be treated aggressively to control hypertension to the lowest tolerated blood pressure. Beta-blockers have the desired effect of reducing the inotropic state of the heart which decreases the impact force of the ejected blood on the aorta resulting to a lower heart rate and blood pressure [12]. For most patients, systolic blood pressure should be controlled between 100 and $120 \mathrm{mmHg}$ with a heart rate of approximately $60 \mathrm{bpm}$. Medical therapy to control pain and decrease lipids is also essential in all patients. Smokers, such as in our patient, have twice the aneurysm expansion rate of nonsmokers and higher dissection rates; hence are advised smoking cessation [13].

In this case report, we describe an elderly patient with a thoracic aortic aneurysm and aberrant right common carotid artery who was treated with a hybrid procedure wherein a combination of surgery and endovascular methods was done for a less invasive approach, which is the first reported case in this institution. The advances in procedures and device development for TEVAR led to the development of hybrid procedures for aortic arch repair in the last decade. Their common concept involves bypass grafting of the supraaortic branches and ligation of the native branches followed by endovascular stent grafting of the aortic arch with or without subsequent TEVAR of the aorta.

TEVAR has gradually replaced open repair with equal safety and efficacy; and consistently yielded lower rates of early mortality and common postoperative complications than open repair. One study reports perioperative mortality rates of $2.1 \%$ versus $11.7 \%$, spinal cord ischemia rates of $3 \%$ versus $14 \%$, respiratory failure rates of $4 \%$ versus $20 \%$, and renal insufficiency rates of $1 \%$ versus $13 \%$ in low-risk patients after endovascular and open repair, respectively. TEVAR carries the additional risk of embolic stroke due to the passage of endovascular wires, catheters, and other devices through the aortic arch; nevertheless the rates are minimal [14]. These complications were not seen in our patient.

The type of approach used should be tailored to the anatomic variables associated with the aortic anomalies. The technical challenges of stent graft deployment, such as proximity to the great vessels and arch tortuosity, have been and remain a focus of device engineering [15].

Aside from CT angiogram, a conventional CT aortogram was done in our patient to better visualize the aberrant vessels along with its blood flow along its critical supra-aortic branches. Detailed evaluation of the aortic arch anatomy is important for planning thoracic surgery and endovascular intervention since the presence and pattern of arch variants and anomalies may influence the surgical incision, cardiopulmonary bypass cannulation and interventional approach. In addition, decisions regarding whether to repair coexisting lesions in the same procedure should be considered $[15,16]$.

Both open and hybrid approaches have been advocated when aortic reconstruction is necessary. It may be necessary for aneurysmal degeneration such as in our case. A thorough understanding of the anatomic landmarks and cerebral perfusion is necessary to guide management options. Standard open aortic reconstruction with or without cardiopulmonary bypass has been described historically which is performed through a left thoracotomy or median sternotomy. The operative mortalities in standard open aortic repair has been reported to be as high as $23 \%$ [17]. The introduction of thoracic endografting has changed the available management options avoiding cardiopulmonary bypass and circulatory arrest resulting to better patient outcomes and earlier recovery.

\section{Conclusion}

A 63-year-old male, a known hypertensive for 23 years, was admitted due to a sudden onset of dull back pain with a pain scale $7 / 10$ radiating to the left anterior chest which was not associated with any dyspnea nor diaphoresis. Initial work-up on chest radiography revealed a widened mediastinum compatible with a thoracic aortic aneurysm which was later confirmed in CT angiogram showing an aberrant right common carotid artery with a noted ascending aorta and aortic arch aneurysm, and proximal descending aorta ectasia with perianeurysmal hematoma.

The patient was admitted as a case of a thoracic aortic aneurysm wherein strict blood pressure control was done with a goal of maintaining systolic blood pressure at 100 to $110 \mathrm{mmHg}$. The patient underwent a twostaged hybrid surgery consisting of an aortic debranching followed by a thoracic endovascular repair via minimally invasive approach. This case report emphasizes on the following major points. 
First, immediate diagnosis of an acute aortic syndrome is important. In patients presenting with back pain radiating to the left anterior chest, multiple differential diagnoses can be considered. Hence, a thorough history and physical examination can guide us in arriving at a prompt and definite diagnosis.

Second, once an acute aortic syndrome is highly considered, different imaging modalities are vital to finally confirm the diagnosis. Computed tomography is the most reliable diagnostic tool in patients with acute aortic dissection or ruptured aortic aneurysm. In the case of our patient wherein an incidental note of an aberrant right common carotid was seen, further imaging studies are needed such as a conventional CT aortogram for detailed surgical anatomy with the involved aberrant vessels.

Lastly, the advancement of technology led to the development of TEVAR used in hybrid procedures for aortic arch repair of aberrant aortic vessels in the last decades which was done in our patient. This approach has gradually replaced open repair with equal safety and efficacy with excellent surgical outcomes through a carefully planned operative approach. Hybrid surgery such as in our patient consistently showed lower rates of early mortality and postoperative complications than open repair changing the available management options for thoracic aortic aneurysms complicated with an aberrant aortic anomaly.

In general, the combination of an adequate knowledge on anatomy coupled with the appropriate utilization of available traditional and innovative interventions is substantial to medically and surgically address rare anatomic anomalies, such as in our patient. In this way, patients may see a ray of hope that they may achieve their optimum functioning.

\section{References}

1. Christian Olsson, Stefan Thelin, Elisabeth Ståhle, Anders Ekbom, Fredrik Granath (2006) Thoracic aortic aneurysm and dissection: Increasing prevalence and improved outcomes reported in a nationwide population-based study of more than 14,000 cases from 1987 to 2002. Circulation 114: 2611-2618.

2. Mathur A, Mohan V, Ameta D, Gaurav B, Haranahalli $P$ (2016) Aortic aneurysm. J Transl Int Med 4: 35-41.

3. Kuzmik GA, Sang AX, Elefteriades JA (2012) Natural histo- ry of thoracic aortic aneurysms. J Vasc Surg 56: 565-571.

4. Kumar A, Kumar K, Zeltser R, Makaryus AN (2016) Nearly asymptomatic eight-month thoracic aortic dissection. Clinical Medicine Insights Cardiology 10: 75-78.

5. Raimund Erbel, Victor Aboyans, Catherine Boileau, Eduardo Bossone, Roberto Di Bartolomeo, et al. (2014) 2014 ESC guidelines on the diagnosis and treatment of aortic diseases: Document covering acute and chronic aortic diseases of the thoracic and abdominal aorta of the adult. The task force for the diagnosis and treatment of aortic diseases of the European Society of Cardiology (ESC). Eur Heart J 35: 2873-2926.

6. Poon FW, Stewart IS (1988) Aneurysm of an aberrant right subclavian artery. Br J Radiol 61: 249-251.

7. HA Alsaif, WS Ramadan (2010) An anatomical study of theaortic arch variations. JKAU: Med Sci 17: 37-54.

8. S Kadir (1991) Regional anatomy of the thoracic aorta, in Atlas of normal and variant angiographic anatomy. In: S Kadir, WB Saunders, Philadelphia, Pa, USA.

9. Gross RE, Ware PF (1946) The surgical significance of aortic arch anomalies. Surg Gynecol Obstet 83: 435-448.

10. Janssen M, Baggen MG, Veen HF, AJ Smout, JA Bekkers, et al. (2000) Dysphagia lusoria: Manometric findings, diagnosis, and therapy. Am J Gastroenterol 95: 1411-1416.

11. YS Prakash, Joseph F Malouf (2004) Aberrant right common carotid artery: A diagnostic challenge on transesophageal echocardiography. Int J Cardiovasc Imaging 20: 217219.

12. Nienaber CA, Powell JT (2012) Management of acute aortic syndromes. Eur Heart J 33: 26-35.

13. Dapunt OE, Galla JD, Sadeghi AM, SL Lansman, CK Mezrow, et al. (1994) The natural history of thoracic aortic aneurysms. J Thorac Cardiovasc Surg 107: 1323-1332.

14. Bavaria JE, Appoo JJ, Makaroun MS, Joel Verter, Zi Fan $\mathrm{Yu}$, et al. (2007) Endovascular stent grafting versus open surgical repair of descending thoracic aortic aneurysms in low-risk patients: A multicenter comparative trial. J Torac Cardiovasc Surg 133: 369-377.

15. F Setacci, P Sirignano, G de Donato, E Chisci, G Galzerano, et al. (2009) Anomalies and variant anatomy of the aorta and the supra-aortic vessels: Additional challenges met by hybrid procedures. HSR Proc Intensive Care Cardiovasc Anesth 1: 37-44.

16. McElhinney DB, Tworetzky W, Hanley FL, Rudolph AM (1999) Congenital obstructive lesions of the right aortic arch. Ann Thorac Surg 67: 1194-1120.

17. Wong DR, Lemaire SA, Coselli JS (2008) Managing dissections of the thoracic aorta. Am Surg 74: 364-380. 


\section{Appendix}

1. Hematology.

\begin{tabular}{|l|l|l|}
\hline Complete Blood Count & Result & Reference Range \\
\hline Hemoglobin & 127 & $135-175 \mathrm{~g} / \mathrm{L}$ \\
\hline Hematocrit & 0.37 & $0.40-0.52$ \\
\hline RBC count & 4.15 & $4.20-6.10 \times 10.6 / \mathrm{LL}$ \\
\hline WBC count & 12.34 & $5.0-10.0 \times 10.3 / \mathrm{LL}$ \\
\hline Differential Count & & \\
\hline Neutrophil & 84 & $55-75 \%$ \\
\hline Lymphocytes & 9 & $20-35 \%$ \\
\hline Monocytes & 5 & $2-10 \%$ \\
\hline Eosinophil & 2 & $1-8 \%$ \\
\hline Basophil & 0 & $0-1 \%$ \\
\hline Platelet count & 204 & $150-400 \times 10.3 / \mathrm{LL}$ \\
\hline MCH & 88.7 & $25.70-32.20 \mathrm{pg}$ \\
\hline MCHC & 30.6 & $32.30-36.50 \mathrm{~g} / \mathrm{dL}$ \\
\hline MCV & 34.5 & $79.00-92.20 \mathrm{fl}$ \\
\hline
\end{tabular}

\section{Clinical chemistry.}

\begin{tabular}{|l|l|l|}
\hline Calcium & 2.08 & $1.75-2.39 \mathrm{mmol} / \mathrm{L}$ \\
\hline Potassium & 3.1 & $3.6-5.1 \mathrm{mmol} / \mathrm{L}$ \\
\hline Serum Magnesium & 0.79 & $0.74-1.03 \mathrm{mmol} / \mathrm{L}$ \\
\hline Sodium & 145 & $135-145 \mathrm{mmol} / \mathrm{L}$ \\
\hline Creatinine & 81.6 & $39-113 \mathrm{umol} / \mathrm{L}$ \\
\hline Troponin I & $0.02 \mathrm{ng} / \mathrm{ml}$ & $0.00-0.04$ \\
\hline
\end{tabular}

\section{Lipid profile.}

\begin{tabular}{|l|l|l|}
\hline Lipid & Result & Reference Range \\
\hline Cholesterol & 3.96 & $<5.15 \mathrm{mmol} / \mathrm{L}$ \\
\hline Triglycerides & 0.49 & $0.00-1.95 \mathrm{mmol} / \mathrm{L}$ \\
\hline HDL - C & 0.8 & $0.00-1.03 \mathrm{mmol} / \mathrm{L}$ \\
\hline LDL & 2.93 & $0.00-1.48 \mathrm{mmol} / \mathrm{L}$ \\
\hline VLDL & 0.22 & $0.00-0.43 \mathrm{mmol} / \mathrm{L}$ \\
\hline
\end{tabular}

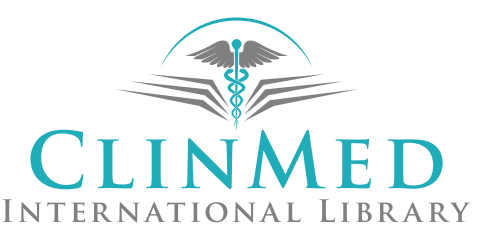

